\title{
Cyanoacrylate glue versus suture fixation of mesh in inguinal hernia open repair: a randomized controlled clinical trial
}

\author{
Abstract \\ Background: The Lichtenstein technique for open hernia repair is associated to a high rate \\ of postoperative chronic groin pain, mainly related to the mesh fixation technique. This \\ randomized controlled trial was aimed to compare the classical suture fixation with glue \\ fixation of the mesh.
}

Methods: Forty-five male patients with primary unilateral groin hernia were randomized to undergo open hernia repair with suture fixation (Group A) or cyanoacrylate glue fixation of the mesh (Group B). Primary outcome was early and late postoperative pain. Secondary endpoints were use of painkillers after 24 hours, morbidity rate and recurrence rate.

Results: Early postoperative pain and pain between 48 hours and 1 month after surgery were significantly lower in Group B. Only two patients had chronic pain, and both were in Group A. Clinical recurrences were two, both in Group A.

Conclusion: This trial demonstrates that mesh fixation with glue is a safe procedure that causes less early and late postoperative pain than the classical suture fixation in open mesh repair of groin hernias.
Volume 2 Issue 5 - 2015

\author{
Giovanni Domenico Tebala,' Valeria Tognoni, ${ }^{2}$ \\ Zoe Tristram,' Francesca Macciocchi, ${ }^{3}$ Paolo \\ Innocenti ${ }^{4}$ \\ 'Department of Surgery, Noble's Hospital, Isle of Man \\ ${ }^{2}$ General Surgery Department, TorVergata University Hospital, \\ Italy \\ ${ }^{3}$ Psychology Department, La Sapienza University, Italy \\ ${ }^{4}$ General Surgery Department, Aurelia Hospital, Italy
}

\section{Correspondence: Giovanni D Tebala, Consultant}

Gastrointestinal Surgeon, Noble?s Hospital, Strang, Douglas, Isle of Man IM4 4RJ, Tel 44I624650207, Fax 447624436I39 Email giovanni.tebala@gov.im

Received: June 15,2015 | Published: September 04, 2015

Keywords: inguinal hernia, hernia repair, cyanoacrylate glue, mesh fixation

\section{Introduction}

The Lichtenstein technique is currently the first choice to repair unilateral primary groin hernias. ${ }^{1}$ [Despite the demonstration of low morbidity and good long term results, ${ }^{2}$ several recent articles demonstrated an unacceptable high rate of chronic inguinal pain, with an average incidence of $12 \%$, but sometimes reported as high as $53 \% .^{3-}$ ${ }^{11}$ Many studies considered chronic postoperative pain as a surgical primary outcome ${ }^{9}$ and few of them evaluated the social impact of post-herniorraphy chronic pain, that has been reported to affect social and work life of up to $6 \%$ of patient. ${ }^{3,5,10,12}$ Considering that some 20 millions hernia repairs are performed each year worldwide, ${ }^{13}$ it necessarily follows that more than 1 million patients worldwide have their lives negatively modified by chronic pain after an "easy" hernia repair. Many factors have been blamed for the development of postherniorraphy chronic pain; i.e. experience of the surgeon, presence of pain even before the operation, surgical technique, mesh fixation. After the introduction of tension-free techniques, chronic groin pain can be due to nerve entrapment in the suture or in the postoperative scar tissue, inflammation of the periosteum of the pubic tubercle traditionally taken into the first stitch, foreign body reaction to the mesh. ${ }^{14,15}$ To avoid these problems and reduce the risk of chronic pain, different methods of mesh fixation have been considered and mainly tissue-compatible glues. The ideal adhesive material should be:
a. Biocompatible
b. Cheap
c. Easy to store and use.

Fibrin glue, even if it gave very good results, ${ }^{16-18}$ does not fulfil the requirements (b) and (c). In our opinion, long-lateral-chain cyanoacrylates represent the best choice for mesh fixation in open mesh repair of inguinal hernia. This randomized controlled clinical trial was designed to evaluate the impact of the mesh fixation method on postoperative pain, and in particular to demonstrate that the use of cyanoacrylate glue can reduce post-herniorraphy pain with respect to the usual method of mesh fixation.

\section{Material and methods}

This randomized controlled trial was carried out at the Aurelia Hospital of Rome, Italy, after approval by the Medical Director and the Local Ethical Committee of the hospital. Final data were discussed and elaborated at the Aurelia Hospital and the Noble's Hospital of the Isle of Man. Male adults with primary unilateral groin hernia suitable for elective open mesh repair were involved in the study and gave informed consent. Exclusion criteria were: bilateral and/or recurrent hernia, femoral hernia, emergency presentation, chronic steroid treatment, coagulation disorders, ongoing chemotherapy, connective tissue disorders and psychological or physical disorders that could affect the ability to feel and elaborate pain.

Primary outcome was early and late postoperative pain. Secondary outcomes were use of painkillers after 24 hours, morbidity rate and recurrence rate. To make the two groups comparable and to reduce the risk of selection bias, all patients underwent a psychological evaluation with the STAI (forms $\mathrm{x}-1$ and $\mathrm{x}-2$ ), TAS20 and the BDI test. The STAI (State-Trait Anxiety Inventory) is a psychological inventory that consists of 40 questions on self-report basis. The STAI measures state anxiety ( $\mathrm{x}-1$, anxiety about a specific event) and trait anxiety ( $\mathrm{x}-2$, anxiety level as a personal feature). Higher scores are positively correlated with higher levels of anxiety. The TAS20 (Toronto Alexithymia scale) is a tool to measure alexithymia, that is the incapacity to identify and describe emotions. It has 20 items, each of them can be rated 1 to 5 . A total score less or equal to 51 means 
no alexithymia, equal or greater than 61 means alexithymia, between 52 and 60 means probable alexithymia. ${ }^{19,20}$ The Beck Depression Inventory is a self-report questionnaire to explore depression. We have used the last version of the BDI, revised in 1996, that contains 21 questions, each being scored 0 to 3 . A total score less than 14 means no or minimal depression, of no clinical significance, 14-19 means mild depression, 20-28 moderate depression, more than 28 means severe depression. ${ }^{21}$ Psychological evaluation has been performed by a psychologist. Patients who were suitable to be entered into the trial (no exclusion criteria) were randomized into one of the two groups:

a. Classical Lichtenstein technique

b. Sutureless Lichtenstein technique with mesh gluing with cyanoacrylate.

Randomization was conducted with the numbered closed envelopes method and occurred at the operation. The first part of the operation was the same in the two groups, according to the original description by Lichtenstein. ${ }^{1}$ Inguinal canal was prepared, alongside with the anatomical landmarks - pubic tubercle, conjoined area, inguinal ligament. The hernia sac was prepared and reduced. The mesh was shaped according to shape and size of the inguinal canal and put in place. In Group A the mesh was fixed with two running sutures both starting from the first stitch passed on the tissue above the pubic tubercle (avoiding the periosteum and with a $2 \mathrm{~cm}$ overlap of the mesh above the tubercle) and passed on the conjoined area and the inguinal ligament. The two posterior wings of the mesh were sutured together with two single prolene stitches. In Group B the mesh was fixed with n-butyl-2-cyanoacrylate tissue adhesive on the pubic tubercle, the inguinal ligament and the conjoined area. Attention was paid to avoid dripping the glue on the nerves. Only one vial of glue was used for each patient. The two posterior wings of the mesh were stitched with a single vicryl stitch paying attention to take only the mesh and not any tissue. All patients had the same polypropylene kind of mesh, irrespective of the fixation method. The fascia was closed in both groups with a vicryl running suture. Skin was closed with a subcuticular vicryl running suture. Nerves were never prepared or cut, in either group.
All operations were performed with spinal block. No postoperative analgesic device was used. Postoperative analgesic treatment was just intramuscular ketorolac upon request of the patient when patient was still in hospital, as required oral paracetamol was prescribed after discharge. Postoperative pain was measured with VAS by direct interview or by phone call at 3 hours, 24 hours, 48 hours, 7 days, 15 days, 1 month, 3 months and 6 months after the operation by a psychologist who was blinded to the treatment allocation. Clinical check was done at 1 month and 6 months by a surgeon who was blinded to the treatment the patient received. According to Alfieri et al. ${ }^{22}$ chronic pain was considered pain at the surgical site still present at the 6th month follow-up. Data were anonymised and stored and elaborated in an electronic database. Data are reported as mean \pm standard deviation or as raw number and percentage for descriptive purposes. Statistical comparison was conducted with the MannWhitney test for independent samples for ordinal and continuous variables and the Chi-square test for proportions. $\mathrm{P}<0.05$ was considered significant. Non-parametric tests were used instead of usual parametric tests for the low number of cases in each group and the non-normal distribution of data.

\section{Results}

The trial has been suspended before reaching the target number of 100 participants due to evident clinical superiority of one treatment with respect to the other one. This decision was based on a general impression of all the surgeons involved in the study. In particular, the "glue" technique was considered easier and quicker. At postoperative follow-up it was clear that some patients had a smoother course, in terms of less pain and quicker return to their normal activities. For these reasons, four months after the beginning of the study data were analysed and results discussed. Decision was agreed to suspend the study due to evident superiority of one technique with respect to the other. Forty-five patients were finally included in the trial. Nineteen patients were randomly allocated to Group A (suture), 26 to group B (glue). Patients' demographics and preoperative clinical details are listed in Table 1.

\section{Table I Demographics}

\begin{tabular}{|c|c|c|c|}
\hline & Group A (Suture) & Group B (Glue) & $p$ \\
\hline N. of patients & 19 & 26 & 0.086 \\
\hline Age & $42.4 \pm 12.0$ & $47.6 \pm 12.3$ & 0.370 \\
\hline \multicolumn{4}{|l|}{ Type of hernia (Nyhus) $)^{36}$} \\
\hline I & 0 & 1 & \\
\hline 2 & 0 & 3 & \\
\hline $3 a$ & 6 & 4 & \\
\hline $3 b$ & 12 & 17 & \\
\hline 4 & 1 & 1 & \\
\hline Surgeon & & & 0.741 \\
\hline Registrar / Trainee & 2 & 2 & \\
\hline Experienced surgeon & 17 & 24 & \\
\hline Psychological evaluation & & & 0.423 \\
\hline STAI $(x-1)$ & $41.8 \pm 8.3$ & $4 I .5 \pm 6.1$ & 0.099 \\
\hline STAI $(x-2)$ & $36.8 \pm 5.4$ & $34.9 \pm 6.7$ & 0.182 \\
\hline TAS20 & $46.2 \pm 8.4$ & $49.5 \pm 10.9$ & 0.214 \\
\hline BDI & $7.6 \pm 6.4$ & $6.0 \pm 3.5$ & \\
\hline
\end{tabular}


The two groups were matched for age, psychological behaviour and type of hernia. Only two patients each group were operated on by trainees. Table 2 shows the course of postoperative pain and compares the two groups. Postoperative pain had a peak after 24 hours, and then decreased progressively. The two curves of pain showed a similar trend, but Group B curve (patients whose mesh was fixed with glue) showed a lower level of pain. In particular, early postoperative pain and pain between 48 hours and 1 month after the operation were significantly lower in Group B. In postoperative day (POD) 1, pain score was similar in the two groups. Long term pain shows a clear difference, but this is not statistically significant maybe due to the low number of cases. Very few patients had painkillers after POD1; 3 in Group A (15.8\%) and 2 in Group B (7.7\%), but this difference is not significant. There was no intraoperative complication and no mortality. General morbidity, including chronic pain was $21.1 \%$ in Group A and 3.8\% in Group B. This difference shows a clear trend but it is still not statistically significant due to the low number of cases. However, morbidity was not clinically significant. In Group A one patient had superficial surgical site infection, one had a seroma, two had chronic pain and one had urinary retention (one patient had two complications). In Group B one patient had urinary retention. No major complications, no reoperation and no infection of the mesh occurred in either group. Two cases in Group A and none in Group B had still pain 6 months after surgery. Also this difference shows a clear trend but is not statistically significant. Clinical recurrences were 2 in Group A and none in Group B. Also this difference is not statistically significant but $\mathrm{p}$-value is low.

Table 2 Postoperative pain measured by VAS

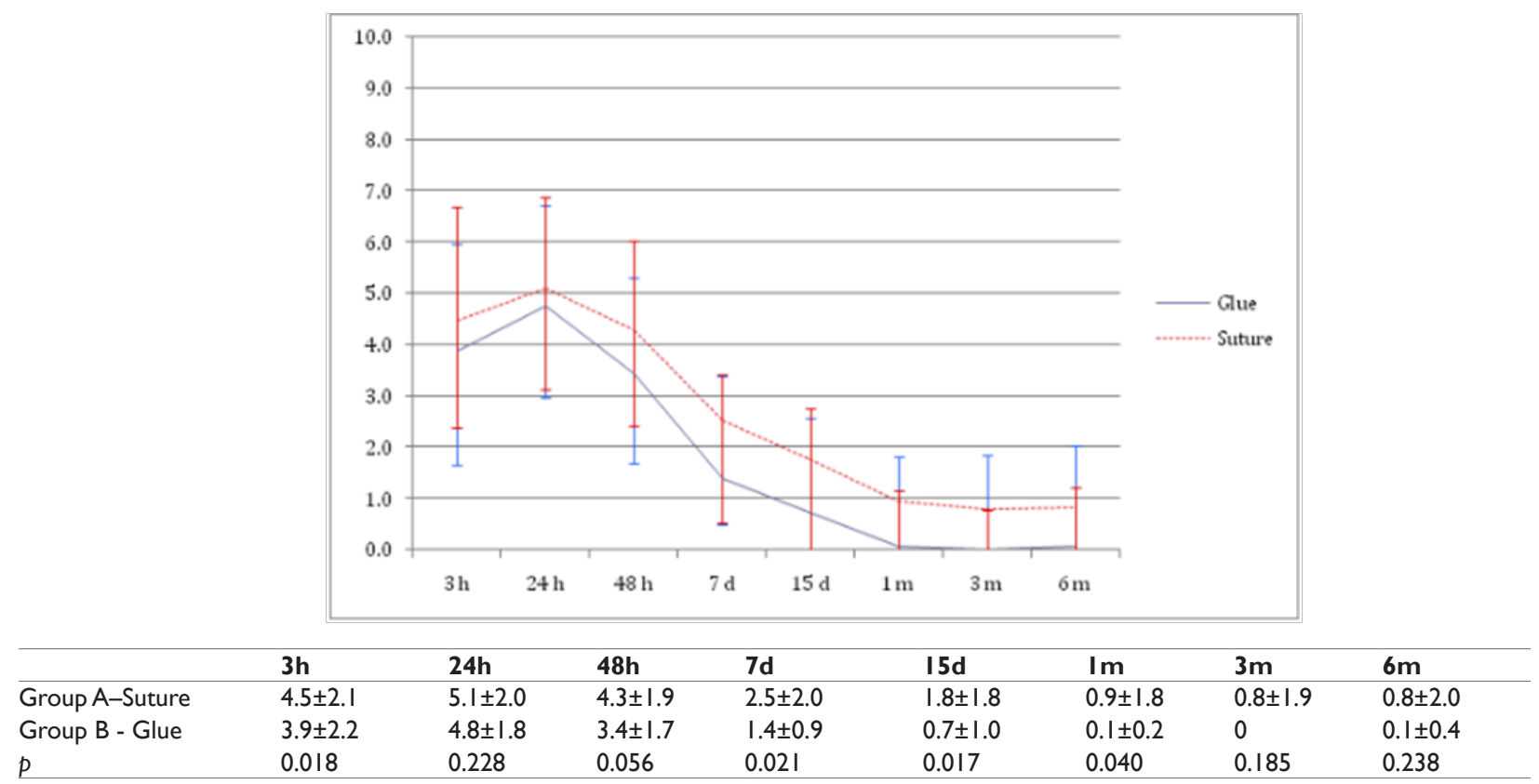

\section{Discussion}

Open mesh repair of primary unilateral inguinal hernia is one of the most frequent operations worldwide. ${ }^{3}$ It is a straightforward operation in most cases, and is one of the first operations a surgical trainee performs without supervision. Nevertheless, it is cause of chronic pain, discomfort and reduced quality of life in an unacceptable number of cases. ${ }^{4-12}$ The method of fixation of the mesh is one of the factors that have been blamed for postoperative pain. Sutures, mostly nonabsorbable, have been demonstrated to increase postoperative pain by nerve entrapment or injury or chronic foreign body reaction. ${ }^{14,15}$ Different methods of mesh fixation have been studied. Self-gripping meshes have been introduced quite recently and their use is still not diffuse, so currently they can be considered only a possible alternative, should their costs match those of "normal" meshes. ${ }^{23,24}$

The use of tissue adhesives has had the most promising results in terms of clinical efficacy and reduction of postoperative pain. ${ }^{25}$ The ideal adhesive material should be:

1. Biocompatible

2. Cheap

3. Easy to store and use.
Despite its very good results, ${ }^{16-28,26}$ fibrin glue does not fulfil the requirements (b) and (c). In our opinion, mid or long-lateral-chain cyanoacrylates represent the best choice for mesh fixation in open mesh repair of inguinal hernia. In the presence of a lateral chain made by a methyl or ethyl radical, the polymerization of cyanoacrylate is too exothermic, with local production of high temperature. This can cause tissue injury and also systemic problems, but they are currently used in industry and everyday life. On the contrary, butyl- and octylcyanoacrylates demonstrated very good tissue compatibility and have been used extensively in medicine and surgery. Cyanoacrylate tissue adhesive is quite cheap, with respect to fibrin glue, it is easily stored even for long time and can be easily applied. This prospective randomized clinical trial was aimed at comparing suture mesh fixation with glue mesh fixation in Lichtenstein open hernia repair. Unfortunately, some of the figures did not reach statistical significance due to the low number of cases. In fact, it was impossible to reach the programmed number of cases, due to the evident clinical superiority of glue with respect to suture fixation. A few months from starting the trial, the general feeling among the surgeons was that patients whose mesh was fixed with glue had less postoperative pain and quicker return to their normal activity. Moreover, operative time was shorter. For these reasons it was considered unethical to continue with the trial. The results were analysed and conclusions drawn. 
Unfortunately, operative time was not an endpoint of the study, so it was not recorded and no data are provided to support the assumption of a shorter surgical time in glued mesh hernia repair. However, the first impression and mostly the results of this pilot study convinced us of the superiority of glue vs suture in the fixation of mesh and we changed our practice as a result of this. Nowadays, we almost always use to fix our meshes with cyanoacrylate glue, both in open and laparoscopic hernia repair. Primary endpoint of our trial was early and late postoperative pain. According to Alfieri et al. ${ }^{22}$ chronic pain has been defined as the pain lasting 6 or more months after the operation.

In our study, the two curves of pain had similar trend but different levels of pain score. The peak of pain was in both groups 24 hours after surgery, when the effect of intraoperative anaesthesia had completely vanished and patient had restarted his normal life. At this stage there was no difference between glue and suture, and maybe pain was mostly due to the surgical trauma itself and the local release of inflammatory mediators. Also the number of patients who were still on painkillers after 24 hours is comparable between the two groups. This is quite different from the figures reported in some meta-analyses that demonstrated also a reduction of acute pain with glue fixation. ${ }^{25,27}$ After that, pain gradually decreased to reach a plateau 1 month after surgery. Patients who have still pain 3 months after surgery most likely will have chronic pain. The most significant differences between the two groups are between 2 days and 1 month after surgery, maybe due to the low-grade local inflammation and reduced risk of nerve entrapment when glue was used. But there is an interesting trend also in the long run, as the only two patients who still had pain at the 6th month follow-up visit were in group A. However, the difference of average pain between the two groups is not significant at 3 and 6 months after the operation. In this respect, RCTs by Dabrowiecki et al. ${ }^{28}$ \& Paajanen et al. ${ }^{29}$ failed to find any difference in long term pain. In this last study, however, the control group was of patients whose mesh was fixed with few absorbable stitches and not the classic polypropylene running suture. This can account for the totally overlapping results. Some recent meta-analyses and systematic reviews confirmed a reduction in chronic groin pain and a faster return to normal activities with the use of glue, ${ }^{25}$ whereas others did not reach the same conclusions. ${ }^{30,31}$ Duration of time to return to normal activities was not an endpoint of our study. With a longer follow up, Kim-Fuchs et al. have been able to demonstrate a trend for less pain in the glue group with respect to the suture group up to 5 years after surgery. ${ }^{32}$ This indicates that chronic pain is no more linked to local inflammation or micro environmental changes, but should be due to more anatomical reasons, such as nerve entrapment. However, this has not been confirmed in meta-analysis studies. ${ }^{27}$

Secondary endpoints were postoperative complications and recurrence rate; in our case both of them seem to be higher in the group of patients whose mesh was fixed with suture. The specific analysis of the single complications shows that none of them can theoretically be related to the fixation method. In 2010 Testini et al. reported an overall morbidity rate as high as $39 \%$ in "suture" patients, vs $11 \%$ in "cyanoacrylate" patients. ${ }^{33}$ This is quite impressive, but if we analyse the single complications, we can gather that the most of this difference is due to nervous complications (pain and numbness). If we don't consider those events, morbidity rates come down to $6.8 \%$ vs $1.8 \%$ ( $p=$ not significant). Assuming that fixation with two polypropylene running sutures is somehow the best guarantee against mesh slippage, we have not been able to understand the real causative factors of the higher recurrence rate in Group A. It is likely that those two recurrences are due to technical failure more than to the suture fixation of the mesh. Anyway, this figure contrasts with those reported by Kim-Fuchs et al., who found a recurrence rate in patients whose mesh was fixed with glue almost double than that in patient whose mesh was sutured. ${ }^{32}$ However, it has been demonstrated that cyanoacrylate glue produces more than adequate fixation strength, significantly better than that of fibrin glue. ${ }^{34}$

Many RCTs have been conducted to compare glue fixation with suture fixation. Most of them are of low or very low quality and heterogeneity is high. ${ }^{30}$ More significant results could come from systematic reviews and meta-analyses, but they are sometimes contrasting. ${ }^{22}$ This can be due to the ample heterogeneity of the studies, probably much more than reported in the published articles. In fact, groin hernia surgery is one of the less standardized procedures, as every single step of a "simple" operation as the original Lichtenstein technique has been modified by every surgeon. Some variables are skin incision (length, direction), preparation of the fascia (subcutaneous fat left on the fascia vs complete dissection), plication of the transversalis fascia, dissection of the pubic tubercle, treatment of cremasteric fascia, overlap of the mesh on the pubic tubercle, technique of closure only to cite a few. Each of them can affect postoperative pain. It would be interesting to perform a study aimed at exploring the many different techniques of open hernia repair. In our study we planned a standardized technique, trying to limit this bias, but we cannot exclude that occasionally the surgeon performed some "personal" steps to face unexpected intraoperative findings. This is an obvious bias of all the reported RCTs, and of this study in particular.

Clearly, this study has some other limitations. The most evident is the low number of cases. The original research plan was to conduct a pilot study on 100 patients, however this was stopped after four months due to evident better clinical results in the group of patients whose mesh was fixed with glue. This was based on a general impression of all the surgeons involved in the study. The clinical difference between the two groups is only partially demonstrated by the objective indicators hereby reported. The second limitation is the short follow up. During the study-planning it was decided that 6 months follow up could have been a reasonable time as pain still present at 6 months is to be considered "chronic". Our primary endpoint was then achieved. Unfortunately, 6 months could be considered a non-sufficient interval to estimate the recurrence rate, as many of them can occur in the long term. Strength of this study is undoubtedly the preoperative psychological evaluation of patients. In fact, at our knowledge, no previous randomized controlled study matched the two groups also with respect to psychological trait. It is well demonstrated that postoperative pain is affected by psychology ${ }^{35}$ - anxiety, depression and attitude culture, education, personal history, religion and this should be considered a confounding bias in the clinical researches on pain perception. In our study this bias has been eliminated.

\section{Conclusion}

This trial demonstrated once again that mesh fixation with glue causes less postoperative pain-both acute and chronic than the classical suture fixation, with similar if not better morbidity and recurrence rates. A large scale well planned double-blinded randomized controlled trial with a standardized technique and long term follow-up could give more definitive results, in particular if it entails a multivariate analysis to understand the actual weight of each single factor in the genesis of post-herniorraphy chronic pain.

\section{Acknowledgements}

The Authors would like to thank prof. Franco Ceriati, who inspired this study many years ago. 


\section{Conflicts of interest}

The authors declare that there is no conflict of interest.

\section{References}

1. Lichtenstein IL, Shulman AG, Amid PK, et al. The tension-free hernioplasty. Am J Surg. 1989;157(2):188-193.

2. Amid PK, Lichtenstein IL. Long-term results and current status of the Lichtenstein open tension-free hernioplasty. Hernia. 1998;2(2):89-94.

3. Bay-Nielsen M, Kehlet H, Strand L, et al. Quality assessment of 26,304 herniorrhaphies in Denmark: a prospective nationwide study. Lancet. 2001;358(9288):1124-1128.

4. Erhan Y, Erhan E, Aydede H, et al. Chronic pain after Lichtestein and preperitoneal (posterior) hernia repair. Can J Surg. 2008;51(5):383-387.

5. Franneby U, Sandblom G, Nordin P, et al. Risk factors for long-term pain after hernia surgery. Ann Surg. 2006;244(2):212-219.

6. Hetzer FH, Hotz T, Steinke W, et al. Gold standard for inguinal hernia repair: Shouldice or Lichtenstein? Hernia. 1999;3(3):117-120.

7. Kumar S, Wilson RG, Nixon SJ, et al. Chronic pain after laparoscopic and open mesh repair of groin hernia. Br J Surg. 2002;89(11):1476-1479 .

8. Paajanen H. Do absorbable mesh sutures cause less chronic pain than nonabsorbable sutures after Lichtenstein inguinal herniorraphy? Hernia. 2002;6(1):26-28.

9. Perkins FM, Kehlet H. Chronic pain as an outcome of surgery. A review of predictive factors. Anesthesiology. 2000;93(4):1123-1133.

10. Poobalan AS, Bruce J, King PM, et al. Chronic pain and quality of life following open inguinal hernia repair. Br J Surg. 2001;88(8):1122-1126.

11. Poobalan AS, Bruce J, Smith WC, et al. A review of chronic pain after inguinal herniorraphy. Clin J Pain. 2003;19(1): 48-54.

12. Kehlet H. Chronic pain after groin hernia repair. $\mathrm{Br} J$ Surg 2008;95(2):135-136.

13. Bay-Nielsen M, Nilsson E, Nordin P, et al. Chronic pain after open mesh and sutured repair of indirect inguinal hernia in young males. Br J Surg. 2004;91(10):1372-1376.

14. Helbling C, Schlumpf R. Sutureless Lichtenstein: first results of a prospective randomised clinical trial. Hernia. 2003;7(2):80-84.

15. Klosterhalfen B, Klinge U, Hermanns B, et al. Pathologie traditioneller chirurgischer Netze zur Hernienreparation nach langzeitimplantation in Menschen. Chirurg. 2000;71(1):43-51.

16. Campanelli G, Champault G, Pascual MH, et al. Randomized controlled blinded trial of Tissucol/Tisseel for mesh fixation in patients undergoing Lichtenstein technique for primary inguinal hernia repair: rationale and study design of the TIMELI trial. Hernia. 2008;12(2):159-165.

17. Lovisetto F, Zonta S, Rota E, et al. Use of human fibril glue (Tissucol) versus staples for mesh fixation in laparoscopic transabdominal preperitoneal hernioplasty: a prospective, randomized study. Ann Surg. 2007;245(2):222-231.

18. Schwab R, Schumacher O, Junge K, et al. Fibrin sealant for mesh fixation in Lichtestein repair: biomechanical analysis of different techniques. Hernia. 2006;11(2):139-145.

19. Bagby RM, Parker JDA, Taylor GJ. The twenty-item Toronto Alexithymia Scale - I. Item selection and cross-validation of the factor structure. J Psychosom Res. 1994;38(1):23-32.
20. Bagby RM, Taylor GJ, Parker JD. The twenty-item Toronto Alexithymia Scale - II. Convergent, discriminant and concurrent validity. $J$ Psychosom Res. 1994;38(1):33-40.

21. Beck AT, Steer RA, Ball R, et al. Comparison of Beck Depression Inventories -IA and II in psychiatric outpatients. J Person Assess. 1998;67(3):588-597.

22. Alfieri S, Amid PK, Campanelli G, et al. International guidelines for prevention and management of post-operative chronic pain following inguinal hernia surgery. Hernia. 2011;15(3):239-249.

23. Chastan P. Tension free open inguinal hernia repair using an innovative self gripping semi-resorbable mesh. J Minimal Access Surg. 2006;2(3):139-143.

24. Pedano N, Pastor C, Arredondo J, et al. Open tension-free hernioplasty using a novel lightweight self-gripping mesh: medium-term experience from two institutions. Langenbecks Arch Surg. 2012;397(2):291-295.

25. Colvin HS, Rao A, Cavali M, Campanelli G, Amin AI (2013) Glue versus suture fixation of mesh during open repair of inguinal hernia: a systematic review and meta-analysis. World J Surg. 37(10):2282-2292.

26. Negro P, Basile F, Brescia A, et al. Open tension-free Lichtenstein repair of inguinal hernia: use of fibrin glue versus sutures for mesh fixation. Hernia. 2011;15(1):7-14.

27. De Goede B, Klitisie PJ, VanKempen BJ, et al. Meta-analysis of glue versus sutured mesh fixation for Lichtenstein inguinal hernia repair. $\mathrm{Br} J$ Surg. 2013;100(6):735-742.

28. Dabrowiecki S, Piersciński, Szczęsny W. The Glubran 2 glue for mesh fixation in Lichtensstein's hernia repair: a double-blind randomized study. Wideochir Inne Tech Maloinwazyjne. 2012;(2):96-104.

29. Paajanen H, Kössi J, Silvasti S, et al. Randomized clinical trial of tissue flue versus absorbable sutures for mesh fixation in local anaesthetic Lichtenstein hernia repair. Br J Surg. 2011198(9):1245-1251.

30. Sanders DL, Waydia S. A systematic review of randomised control trials assessing mesh fixation in open inguinal hernia repair. Hernia. 2014;18(2):165-176.

31. Ladwa N, Sajid MS, Sains P, et al. Suture mesh fixation versus glue mesh fixation in open inguinal hernia repair: a systematic review and meta-analysis. Int J Surg. 2013;11(2):128-135.

32. Kim-Fuchs C, Angst E, Vorburger S, et al. Prospective randomized trial comparing sutures with sutureless mesh fixation for Lichtenstein hernia repair: long term results. Hernia. 2012;16(1):21-27.

33. Testini M, Lissidini G, Poli E, et al. A single-surgeon randomized trial comparing sutures, N-butyl-2-cyanoacrylate and human fibrin glue for mesh fixation during primary inguinal hernia repair. Can J Surg. 2010;53(3):155-160.

34. Schug-Pass C, Jacob DA, Rittinghausen J, et al. Biomechanical properties of (semi-) synthetic glues for mesh fixation in endoscopic inguinal hernia repair. Hernia. 2013;17(6):773-777.

35. Theunissen M, Peters ML, Bruce J, et al. Preoperative anxiety and catastrophizing: a systematic review and meta-analysis of the association with chronic postsurgical pain. Clin J Pain. 2012;28(9):819-841.

36. Nyhus LM. Individualization of hernia repair: a new era. Surgery. 1993;114(1):1-2. 